Review

\title{
Perspectives on Hydrate Thermal Conductivity
}

\author{
Niall J. English ${ }^{1, *}$ and John S. Tse ${ }^{2}$
}

1 The SEC Strategic Research Cluster and the Centre for Synthesis and Chemical Biology, School of Chemical and Bioprocess Engineering, University College Dublin, Belfield, Dublin 4, Ireland

Department Physics and Engineering Physics, University of Saskatchewan, Saskatoon, Saskatchewan S7N 5E2, Canada; E-Mail: john.tse@usask.ca

* Author to whom correspondence should be addressed; E-Mail: niall.english@ucd.ie.

Received: 28 October 2010; in revised form: 3 December 2010 / Accepted: 6 December 2010 / Published: 10 December 2010

\begin{abstract}
In this review, the intriguing, anomalous behaviour of hydrate thermal conductivity will be described, and progress in performing experimental measurements will be described briefly. However particular attention shall be devoted to recent advances in the development of detailed theoretical understandings of mechanisms of thermal conduction in clathrate hydrates, and on how information gleaned from molecular simulation has contributed to mechanistic theoretical models.
\end{abstract}

Keywords: Hydrate; thermal conductivity; molecular dynamics

\section{Introduction}

The discovery of enormous quantities of natural gas in the form of gas hydrates in sub-oceanic sediments and permafrost has driven significant interest in their use as a resource, as a potential geohazard, their role in the global carbon cycle, and potential influence on climate change [1-4]. Progress in research in these areas depends fundamentally on the availability of high-quality property data and an increased understanding of underlying physics and chemistry governing clathrate hydrates in nature. Gas hydrates, in general, exhibit an anomalous, glasslike behavior of the thermal conductivity at temperature and pressure conditions occurring in nature that distinguishes them from ice and other molecular crystals [5]. The importance of reliable thermal property data for modeling the behavior of gas hydrates in natural systems has been discussed by Sloan [1], Gupta et al., [6] and 
Waite et al. [3] A detailed understanding of the mechanisms leading to this behavior would enhance the underlying knowledge of hydrate physics that may lead to improvements in simulation programs for determining long-term resource recovery potential and the impact of hydrates on climate change. It is thought that the low thermal conductivity is attributable to the phonon-scattering processes from energy transfer between the localized guest rattling modes and the host lattice acoustic modes [7-11]. A detailed understanding of the mechanisms leading to this behavior, along with the characterization of thermophysical properties, would enhance the viability of large-scale methane production from hydrates $[12,13]$. The purpose of this review is to synopsize progress in experimental determination of these properties, and to focus on advances in theoretical understanding of mechanisms underpinning this behavior.

\section{Experimental Characterisation}

Measurement of the thermal properties of relatively pure hydrate requires the use of pressurized equipment to both form the samples in the laboratory and stabilize them during measurement. It is also desirable to reduce the porosity of the sample after formation by compaction in the same pressure vessel: this influences the thermal conductivity critically in the intermediate temperature range, below the Debye temperature (vide infra). Removal of the sample for compaction introduces the possibility of contamination or decomposition of the hydrate during transfer and handling.

The majority of measurements of thermal conductivity reported for hydrates in the literature have been made using a needle probe on samples prepared using granular ice [3,14-17]. However, more recent work has been reported by Huang et al [18] and Rosenbaum et al [19] using a commercially available transient plane source (TPS) technique in double- and single-sided configurations, respectively. The original double-sided configuration was developed by Gustaffson [20]. Considering methane hydrate as an example, the measured values of thermal conductivity for compacted samples at conditions similar to those in natural environments are $0.62 \pm 0.02 \mathrm{~W} / \mathrm{m} \cdot \mathrm{K}^{3}$ using a needle probe, and 0.57 [18] and $0.68 \pm 0.01 \mathrm{~W} / \mathrm{m} \cdot \mathrm{K}$ [19] using the double- and single-sided TPS techniques, respectively. In the Huang et al study [18], hydrate was formed from methane and water that contained a surfactant to assist hydrate formation, while in the study or Rosenbaum et al, [19] pressurized methane gas was put in contact with ice and followed by repeated cooling and heating cycles to induce hydrate formation, followed by mechanical compaction to reduce the porosity to the lowest possible level. The Waite et al investigation [3] also used compaction, but not to the extent as that of Rosenbaum et al, [19] while comparatively little compaction was employed by Huang et al study [18]. For this reason, it is likely that the result of $0.68 \pm 0.01 \mathrm{~W} / \mathrm{m} \cdot \mathrm{K}$ [19] is the most reliable for pure, circa $90 \%$-occupied methane hydrate with the lowest possible level of porosity. Measures of the thermal diffusivity of methane hydrate have also been reported by Kumar et al. [21] and Tuner et al. [22] for porous (uncompacted) samples, and by deMartin, [15] Waite et al., [3] Rosenbaum et al [19] for compacted samples. The thermal diffusivity values do not seem to be dependent on the presence of some porosity and are in the range of $2-3.1 \times 10^{-7} \mathrm{~m}^{2} / \mathrm{s}^{2}$

The needle probe or double-sided TPS methods need the probe to be surrounded by the hydrate. Although viable in a laboratory environment, such technology may not be easily adapted for in-situ determination of the thermal properties of hydrate or consolidated hydrate/sediment samples. In comparison, the single-sided TPS technique is more conducive towards adaptation for field use in 
hydrate-containing sediments and situations where sample penetration, disturbance and/or immersion of the sensor would be difficult.

Earlier measurements by Ross and Anderson in the early 1980's established that clathrate hydrates exhibit a glass-like temperature dependence of thermal conductivity at temperature and pressure conditions prevalent in nature, in which there is a very weak temperature dependence [14]; the thermal conductivity is also markedly lower than ice, and is in the $0.45-0.7 \mathrm{~W} / \mathrm{m} \cdot \mathrm{K}$ range for $265-280 \mathrm{~K}$ depending on the hydrate guest and sample porosity, vis-à-vis approximately $2.2 \mathrm{~W} / \mathrm{m} \cdot \mathrm{K}$ at $273 \mathrm{~K}$ for ice [23-25]. Other experimental studies since then, such as those mentioned above, have confirmed this trend. Such intriguing behaviour is anomalous for a crystalline solid (vide infra), especially given that other physical properties of hydrates are relatively similar to ice Ih [1,2]. Krivchikov et al have also considered proton ordering effects on the thermal conductivity of THF hydrates [26]. Recently, however, experimental measurements of thermal conductivity in both methane and xenon hydrates by Krivchikov et al led to the highly interesting finding of crystal-like temperature dependence below $90 \mathrm{~K}$, with glass-like behaviour above this temperature [16,17]. The remainder of this review will be focussed on outlining progress in exploring possible mechanisms to rationalise these experimental observations.

A particular difficulty surrounding the direct, quantitative comparison of thermal conductivity from molecular simulation with experimental results is that the experimental determination is highly dependent on samples' nature and quality, e.g., level of porosity, which has been outlined in some detail for methane hydrates $[3,16,19]$. Further problems may well arise with the quality of the potential models used in molecular simulation, in addition to the system size and electrostatics [19].

\section{Mechanisms and Pressure Dependence}

In addition to clathrate hydrates, anomalous "glasslike" thermal conductivity behaviour is shared by some clathrate-like compounds, e.g., metal-Si clathrates [27] and skutterudites [28]. Several hypotheses have been suggested to explain this observation, the most studied being the "resonant scattering" model [7-11,29,30], which originates in the case of hydrates from "avoided-crossings" of lattice acoustic phonons with localized guest vibrations of identical symmetry dissipating heat transport $[10,11,29]$. Similar behaviour to gas hydrates in thermal conductivity, with crystal- and glass-like temperature dependence at low and higher temperatures, respectively, has also been observed in some semi-conductor clathrates [31]. Krivchikov et al have also considered how resonant phonon scattering explains contributions from internal molecular modes towards thermal conductivity in molecular glasses [32].

Prior to discussing advances in theoretical and molecular modelling activities to gain insight into mechanisms governing the behaviour of hydrate thermal conductivity, it is worthwhile making some general remarks about crystalline thermal conductivity behaviour. A crystal's thermal conductivity's temperature profile is well-known. Above half of the Debye temperature, $\Theta_{\mathrm{D}} / 2$, the thermal conductivity exhibits a $T^{-1}$ dependence, explained by Umklapp processes scattering phonons [33,34]. (For methane hydrate, $\Theta_{\mathrm{D}}$ is $226 \mathrm{~K}$ ) Thermal conductivity increases as temperature decreases below $\Theta_{\mathrm{D}} / 2$, reaching a maximum near $\Theta_{\mathrm{D}} / 10$. In this "intermediate" régime, the thermal conductivity's absolute value is heavily dependent on defects. Below around $\Theta_{\mathrm{D}} / 10$, conductivity has $T^{3}$ dependence, governed mainly by boundary scatterings. Therefore, experimental determination of thermal 
conductivity is highly dependent on samples' nature and quality, which has been discussed in some detail for methane hydrates $[3,16,19]$. This underlines the difficulty in a quantitative comparison of theoretical and experimental values, as previous joint experimental-simulation research for methane hydrate has shown [19]. It should also be noted that classical theory is expected to be valid at temperatures above one-tenth of the Debye temperature $\left(\Theta_{\mathrm{D}} / 10\right)$, i.e., approximately $20 \mathrm{~K}$ for (sI) methane hydrate. It is to be expected that amorphous molecular systems, such as amorphous ices, would exhibit very limited temperature dependence in their thermal conductivity, and this been found for higher-density amorphous ices, from both experiment and molecular simulation [36].

In terms of pressure effects on hydrate thermal conductivity, Safarov and Zaripova [37] have studied the conductivity of hydrazine hydrate and found that increasing pressure led to an increased conductivity in the 0.1 to $49 \mathrm{MPa}$ pressure range, increasing by some $15 \%$ from lowest to highest pressures for any given temperature. Anderrson and Johari [38,39] have studied the variation of thermal conductivity of THF hydrate, ice and ice clathrates due to pressure-induced collapse: although increasing pressure led to ice and ice clathrate thermal conductivity decreasing upon collapse, it generally increased for THF hydrate. For THF hydrate conductivity studies measured at temperatures in the range $55-250 \mathrm{~K}$ and at pressures up to $0.16 \mathrm{GPa}$, distinct variation in conductivity with pressure was observed for a proton-ordered low-temperature (LT) and a proton-disordered high-temperature (HT) phase: the LT phase exhibited a slight increase with pressure, whereas the HT phase was largely independent of pressure [38]. In more recent high-pressure studies for pure THF hydrate, they found a slight increase in conductivity at $130 \mathrm{~K}$ with increasing pressure up to $0.75 \mathrm{GPa}$, and a levelling off thereafter up to $0.95 \mathrm{GPa}$, followed by an increase during amorphisation up to $1.25 \mathrm{GPa}$ [39]. This change in thermal conductivity upon a difference in higher-pressure structure has been found also by English et al [36] using molecular simulation for sI and higher-pressure sII and sH polymorphs of methane hydrate.

\section{Theoretical and Molecular Modelling}

Krivchikov et al have analysed the previously measured thermal conductivities of $\mathrm{Xe}, \mathrm{CH}_{4}$, and THF hydrates in a wide region of temperatures [40], for which $\kappa(T)$ at temperatures in natural environments depends weakly on chemical composition, crystal structure and microstructure. The results have been discussed in the context of phenomenological models of phonon scattering by local modes [32], the so-called soft-potential model, building on earlier concepts of resonance scattering [7-11,29,30]. The glass-like behaviour of experimental thermal conductivity data in both sI and sII hydrates at low temperatures in the context of the soft-potential model yields behaviour in $\kappa(T)$ similar to molecular glasses, as studied earlier [32]. It was found in the framework of this model that the anharmonicity of guest motions becomes a dominant factor at lower temperatures in determining heat transfer processes in clathrate structures.

Given the increase in computing power in the past decade or so, it has now become possible to examine heat conduction processes in hydrates using molecular dynamics as a valuable tool to gain insight into the underlying mechanisms, so as to assess important contributing factors to the thermal conductivity and its behaviour. One method for the estimation of thermal conductivity is to calculate it from Green-Kubo (GK) linear response theory [41] via the heat flux autocorrelation function (HCACF) using equilibrium molecular dynamics (MD) [42]. This method has been applied to $\mathrm{Xe}^{8}$ and 
methane hydrates [19,43-46] and to metallic germanium clathrate [47]. Non-equilibrium MD has also been used to predict thermal conductivity of methane hydrate $[48,49]$ and xenon, and carbon dioxide hydrates [49]. Important technical points to consider in MD simulation include assessing if the simulation box is sufficiently large and accurate treatment of long-range electrostatic interactions $[19,43,48]$. It is only relatively recently that it has been recognised that truncations of long-range electrostatic interactions in charged systems lead to errors in the stress tensor [50,51].

Jiang et al have employed non-equilibrium MD to estimate the thermal conductivity of methane, xenon, and carbon dioxide hydrates, using both polarisable and fixed-charge water potentials from 30 to $260 \mathrm{~K}$. [48,49] The calculations were found to match the temperature trend in the experimental data at temperatures below $50 \mathrm{~K}$, but they showed a slight decrease in thermal conductivity at higher temperatures, opposite to the trend in experimental data. It was found for methane hydrate that the calculated thermal conductivity values were relatively insensitive to cavity occupancy except at or below $50 \mathrm{~K}$. This finding was interpreted as an indication that the differences between the lattice structures of methane hydrate and ice Ih may play a more dominant role than previously considered in rationalising the low thermal conductivity of methane hydrate vis-à-vis ice Ih [48]. The introduction of defects into the lattice in methane hydrate was found to induce a reduction in the thermal conductivity but to have little effect on its temperature dependence [48]. In terms of guest-size effects on hydrate thermal conductivity, the study of Jiang at al [49] on the conductivity of $\mathrm{CH}_{4}, \mathrm{CO}_{2}$ and Xe hydrate found that in these sI structures, the methane hydrate had a larger thermal conductivity than $\mathrm{CO}_{2}$ and Xe from non-equilibrium MD, by some $15-20 \%$, across the 30 to $260 \mathrm{~K}$ temperature range, while comparison of the available experimental data for methane and Xe hydrate led to a similar conclusion (that methane hydrate conductivity is around 20\% higher). Although the details of the guest-host interactions are critical in determining phonon propagation in the lattice, larger guests for a particular hydrate type would generally be expected to reduce the amplitude of energy loss through rattling motion.

English and co-workers have reproduced semi-quantitatively the experimental thermal conductivities of ice Ih, empty sI hydrate and sI, sII and sH methane hydrate using the rigorous GK approach with accurate long-range electrostatics in a series of papers with a variety of polarisable and fixed-charge water models, over the 30 to $260 \mathrm{~K}$ range. [19,40-43] The observed reversal in sI's temperature dependence at $90 \mathrm{~K}$ was predicted. Through comprehensive analysis of heat flux correlation functions, methane's role in heat transport dissipation in sI hydrate above $100 \mathrm{~K}$, and to a lesser extent in sII and $\mathrm{sH}$ above $200 \mathrm{~K}$, was demonstrated clearly. Since the (van der Waals) water-methane interaction was found to be weak, it was found that scattering of thermal phonons is much more efficient at higher temperature $(>100 \mathrm{~K})$ in sI methane hydrate. However, less intimate water-methane contact in the larger cavities in sII and sH hydrates means that the temperature required for significant dissipation in water-methane energy transfer is above $200 \mathrm{~K}$, since larger-amplitude thermal rattling of the methane molecules at higher temperature is needed to bring about the intermittent close guest-host contact required for dissipation in energy transfer in larger cavities. Calculations also showed that a hypothetical empty sI hydrate's thermal conductivity behaviour is similar to ice Ih, except with lower absolute values [44,45]: this was found to be attributable mainly to shorter long-range relaxation times for phonon transport, indicating shorter phonon mean free paths. The results of Jiang et al for empty hydrate are somewhat different, from non-equilibrium MD: this 
predicts that the thermal conductivity of the empty hydrate is close to the fully-occupied case and attributes this behaviour to a reduction of phonon mean free paths $[48,49]$. It is not clear why the equilibrium and non-equilibrium approach differ in these observations, but use of different potential models and previous noting of difficulties in agreement of equilibrium and non-equilbrium methods by Inoue et al [8] need to be borne in mind; in any event, the equilibrium-method results [19,40-43] are in semi-quantitative agreement with experiment, which helps to support its validity.

Following observations that a hypothetical empty sI hydrate's thermal conductivity behaviour is similar to ice Ih, except with lower absolute values [44,45], a similar observation has been made in zeolites, with distortions of $\mathrm{SiO}_{4}$ suggested as the cause [53]. In hydrates, the water framework is much more distorted [53] and therefore the hydrogen bond network is more strained than in ice Ih; English et al conjectured that this would result in the inhibition of long-range modes and spatial localization of energy [44,45]. If correct, this conjecture suggests that low thermal conductivity depends not only on guest-host interactions, but is also related to rigidity of the framework, which echoes the speculation of Jiang et al [48] in terms of the role of differences between the ice Ih and hydrate lattice structures in leading to a lower thermal conductivity in clathrates. This conjecture helps to rationalise the apparent absence of "resonant scattering" in some semi-conductor clathrates and skutterudites and low conductivity in empty silicon clathrate; in particular, skutterudites constitute potential candidates of a new class of engineered efficient thermoelectric materials guided by the principle of phonon glass electron crystals (PGEC) [27,28].

\section{Conclusions}

There has been substantial progress in the past half-decade in the establishment of low-temperature crystal-like behaviour of hydrate thermal conductivity by both experiment and modelling. Progress in the soft potential model and refinements to the resonance scattering picture, coupled with insights from MD simulation, have contributed to improved understanding in the mechanisms underpinning hydrate thermal conductivity. Although compaction of experimental hydrate samples reduces the porosity to the lowest possible level attainable in experimental apparatus, structural defects present in real samples are rarely taken into account in molecular simulations, with the exception of by Jiang et al [48]. A greater understanding of the inclusion of defects on thermal conductivity of hydrate-sediment systems would be highly desirable; this represents a considerable future challenge for molecular simulation, as the geometry of the systems would have to be prepared very carefully, and very large systems would be required.

\section{Acknowledgements}

The authors thank the Ireland-Canada University Foundation and the Royal Irish Academy for research visit funding, and Science Foundation Ireland and the European Social Fund SimBioMa initiative for provision of funding to host a recent CECAM workshop on clathrate hydrate molecular simulation. 


\section{References}

1. Makogon, Y.F. Hydrates of Hydrocarbons; PennWell Books: Tulsa, OK, USA, 1997.

2. Sloan, E.D.; Koh, C.A. Clathrate Hydrates of Natural Gases, 3rd ed.; CRC Press, Taylor \& Francis: Boca Raton, FL, USA, 2007.

3. Waite, W.F.; Stern, L.A.; Kirby, S.H.; Winters, W.J.; Mason, D.H. Simultaneous determination of thermal conductivity, thermal diffusivity and specific heat in sI methane hydrate. Geophys. J. Int. 2007, 169, 767-774.

4. Boswell, R. Resource potential of methane hydrate coming into focus. J. Petrol. Sci. Eng. 2007, 56, 9-13.

5. Tse, J.S.; White, M.A. Origin of glassy crystalline behavior in the thermal-properties of clathrate hydrates-A thermal-conductivity study of tetrahydrofuran hydrate. J. Phys. Chem. 1988, 92, 5006-5011.

6. Gupta, A.; Kneafsey, T.J.; Moridis, G.J.; Seol, Y.; Kowalsky, M.B.; Sloan, E.D. Composite thermal conductivity in a large heterogenous porous methane hydrate sample. J. Phys. Chem. B 2006, 110, 16384-16392.

7. Tse, J.S. Loacalized oscillators and heat-conduction in clathrate hydrates. J. Inclusion Phen. Macro. Chem. 1994, 17, 259-266.

8. Inoue, R.; Tanaka, H.; Nakanishi, K. Molecular dynamics simulation study of the anomalous thermal conductivity of clathrate hydrates. J. Chem. Phys. 1996, 104, 9569-9577.

9. Tse, J.S.; Shpakov, V.P.; Murashov, V.V.; Belosludov, V.R. The low frequency vibrations in clathrate hydrates. J. Chem. Phys. 1997, 107, 9271-9274.

10. Tse, J.S.; Ratcliffe, C.I.; Powell, B.M.; Sears, V.P.; Handa, H.P. Rotational and translational motions of trapped methane. Incoherent inelastic neutron scattering of methane hydrate. J. Phys. Chem. A 1997, 101, 4491-4495.

11. Tse, J.S.; Shpakov, V.P., Belosludov, V.R.; Trouw, F.; Handa, Y.P. Coupling of localized guest vibrations with the lattice modes in clathrate hydrates. Europhys. Lett. 2001, 54, 354-360.

12. MacDonald, G.J. The future of methane as an energy resource. Ann. Rev. Energy 1990, 15, 53-83.

13. Kvenvolden, K.A. Methane hydrate-A major reservoir of carbon in the shallow geosphere. Chem. Geol. 1998, 71, 41-51.

14. Ross, R.G.; Andersson, P.; Bäckström, G. Unusual PT dependence of thermal-conductivity for a clathrate hydrate. Nature 1981, 290, 322.

15. deMartin, B.J. Laboratory Measurements of the Thermal Conductivity and Thermal Diffusivity of Methane Hydrate at Simulated in-situ Conditions; Georgia Institute of Technology: Atlanta, GA, USA, 2001.

16. Krivchikov, A.I.; Gorodilov, B.Ya.; Korolyuk, O.A.; Manzhelii, V.G.; Conrad, H.; Press, W. Thermal conductivity of methane-hydrate. J. Low Temp. Phys. 2005, 139, 693-702.

17. Krivchikov, A.I.; Gorodilov, B.Y.; Korolyuk, O.A.; Manzhelii, V.G.; Romantsova, O.O.; Conrad, H.; Press, W.; Tse, J.S.; Klug, D.D. Thermal conductivity of Xe clathrate hydrate at low temperatures. Phys. Rev. B 2006, 73, 064203.

18. Huang, D.Z.; Fan S.S. Thermal conductivity of methane hydrate formed from sodium dodecyl sulphate solution. J. Chem. Eng. Data 2004, 49, 1479-1482. 
19. Rosenbaum, E.J.; English, N.J.; Johnson, J.K.; Shaw, D.W.; Warzinski, R.P. Thermal conductivity of methane hydrate from experiment and molecular simulation. J. Phys. Chem. B 2007, 111, 13194-13205.

20. Gustafsson, S.E. Transient plane source techniques for thermal-conductivity and thermaldiffusivity measurements of solid materials. Rev. Sci. Instruments 1991, 62, 797-804.

21. Kumar, P.; Turner, D.; Sloan, E.D. Thermal diffusivity measurements of porous methane hydrate and hydrate-sediment mixtures. J. Geophys. Res. 2004, 109, B01207.

22. Turner, D.J.; Kumar, P.; Sloan, E.D. A new technique for hydrate thermal diffusivity measurements. Int. J. Thermophys. 2005, 26, 1681-1691.

23. U.S. Army Corps of Engineers. Engineering and Design: Ice Engineering; University Press of the Pacific: Honolulu, HI, USA, 2002; p. 377.

24. Lunardini, V.J. Heat Transfer in Cold Climates; Van Nostrand Reinhold: New York, NY, USA, 1981.

25. Slack, G.A. Thermal-Conductivity of Ice. Phys. Rev. B 1980, 22, 3065-3071.

26. Krivchikov, A.I.; Romantcova, O.O.; Korolyuk, O.A. The effect of proton ordering on the thermal conductivity of tetrahydrofuran clathrate hydrate. Low. Temp. Phys. 2008, 34, 648-654.

27. Slack, G.A. Design concepts for improved thermoelectric materials. In Thermoelectric Materials-New Directions and Approaches; Tritt, T.M., Kanatzidis, M.G., Lyon, H.B., Mahan, G.D., eds.; Materials Research Society: Warrendale, PA, USA, 1997.

28. Nolas, G.S.; Morelli, D.T.; Tritt, T.M. Skutterudites: A phonon-glass-electron crystal approach to advanced thermoelectric energy conversion applications. Ann. Rev. Mater. Sci. 1999, 29, 89-116.

29. Baumert, J.; Gutt, C.; Shpakov, V.P.; Tse, J.S.; Krisch, M.; Müller, M.; Requardt, H.; Klug, D.D.; Janssen, S.; Press, W. Lattice dynamics of methane and xenon hydrate: Observation of symmetryavoided crossings by experiment and theory. Phys. Rev. B 2003, 68, 174301.

30. Tse, J.S.; Klug, D.D.; Zhao, J.Y.; Sturhahn, W.; Alp, E.E.; Baumert, J.; Gutt, C.; Johnson, M.R.; Press, W. Anharmonic motions of $\mathrm{Kr}$ in the clathrate hydrate. Nat. Mater. 2005, 4, 917-921.

31. Cohn, J.L.; Nolas, G.S.; Fessatidis, V.; Metcalf, T.H.; Slack, G.A. Glasslike heat conduction in high-mobility crystalline semiconductors. Phys. Rev. Lett. 1999, 82, 779-782.

32. Krivchikov, A.I.; Yushchenko, A.N.; Korolyuk, O.A.; Bermejo, F.J.; Fernandez-Perea, R.; Bustinduy, I.; Gonzalez, M.A. Effects of resonant phonon scattering from internal molecular modes on the thermal conductivity of molecular glasses. Phys. Rev. B 2008, 77, 024202.

33. Tritt, T.M. Thermal Conductivity: Theory, Properties, and Applications; Kluwer Academic, Plenum Publishers: London, UK, 2004.

34. McGaughey, A.J.H.; Kaviany, M. Phonon transport in molecular dynamics simulations: formulation and thermal conductivity prediction. Adv. Heat Transfer 2006, 39, 169-225.

35. Tse, J.S. Thermal-expansion of the clathrate hydrates of ethylene oxide and tetrahydrofuran. J. Phys. 1987, 48, 543-549.

36. English, N.J.; Tse, J.S.; Gallagher, R. Thermal conductivity in amorphous ices from molecular dynamics. Phys. Rev. B 2010, 82, 092201, and references therein.

37. Safarov, M.M.; Zaripova, M.A. Experimental study of the thermal conductivity of hydrazine hydrate at high values of the state parameters. Measure. Tech. 1993, 36, 435-438. 
38. Andersson, O.; Suga, H. Thermal conductivity of normal and deuterated tetrahydrofuran clathrate hydrates. J. Phys. Chem. Solids 1996, 57, 125-132.

39. Andersson, O.; Suga, H. Pressure-induced collapse of ice clathrate and hexagonal ice mixtures formed by freezing. J. Chem. Phys. 2009, 131, 114503.

40. Krivchikov, A.I.; Korolyuk, O.A.; Romantsova, O.O. Heat transfer in crystalline clathrate hydrates at low temperatures. Low Temp. Phys. 2007, 33, 612-616.

41. McQuarrie, D.A. Statistical Mechanics, 2nd ed.; University Science Books: Sausalito, CA, USA, 2000.

42. Allen, M.P.; Tildesley, D.J. Computer Simulation of Liquids; Oxford University Press: New York, NY, USA, 1989.

43. English, N.J. Effect of electrostatics techniques on the estimation of thermal conductivity via equilibrium molecular dynamics simulation: application to methane hydrate. Mol. Phys. 2008, 106, 1887-1898.

44. English, N.J.; Tse, J.S. Mechanisms for thermal conduction in methane hydrate. Phys. Rev. Lett. 2009, 103, 015901.

45. English, N.J.; Tse, J.S.; Carey, D. Mechanisms for thermal conduction in various polymorphs of methane hydrate. Phys. Rev. B 2009, 80, 134306.

46. English, N.J.; Tse, J.S. Guest and host contributions towards thermal conduction in various polymorphs of methane hydrate. Comput. Mat. Sci. 2010, 49, S176-S180.

47. Dong, J.J.; Sankey, O.K.; Myles, C.W. Theoretical study of the lattice thermal conductivity in Ge framework semiconductors. Phys. Rev. Lett. 2001, 86, 2361-2364.

48. Jiang, H.; Myshakin, E.; Jordan K.D.; Warzinski, R.P. J. Phys. Chem. B 2008, 112, 10207.

49. Jiang, H.; Jordan, K.D. Comparison of the properties of xenon, methane and carbon dioxide hydrates from equilibrium and nonequilibrium molecular dynamics simulations. J. Phys. Chem. C 2010, 114, 5555-5564.

50. Galamba, N.; Nieto de Castro, C.A.; Ely, J.F. Thermal conductivity of molten alkali halides from equilibrium molecular dynamics simulations. J. Chem. Phys. 2004, 120, 8676-8682.

51. Galamba, N.; Nieto de Castro, C.A.; Ely, J.F. Molecular dynamics simulation of the shear viscosity of molten alkali halides. J. Phys. Chem. B 2004, 108, 3658-3662.

52. McGaughey, A.J.H.; Kaviany, M. Thermal conductivity decomposition and analysis using molecular dynamics simulations_-Part II. Complex silica structures. Int. J. Heat Mass Transfer 2004, 47, 1799-1816.

53. Gutt, C.; Asmussen, B.; Press, W.; Johnson, M.R.; Handa, Y.P.; Tse, J.S. The structure of deuterated methane hydrate. J. Chem. Phys. 2000, 113, 4713-4721.

(C) 2010 by the authors; licensee MDPI, Basel, Switzerland. This article is an open access article distributed under the terms and conditions of the Creative Commons Attribution license (http://creativecommons.org/licenses/by/3.0/). 